\title{
Damping torsional oscillations using a SIMO static VAR controller
}

\author{
S. Lee \\ C.-C. Liu
}

Indexing terms: Oscillations, VAR controller, Fast Fourier transform

\begin{abstract}
Stabilising torsional oscillations using a single input multiple output (SIMO) static VAR controller is investigated. An eigenstructure assignment technique is applied to design it. The designed controller can be easily implemented by a multiple proportion integral (MPI) controller using medium pressure turbine speed, two low pressure turbine speeds, and generator shaft speed as the feedback signals. Eigenvalue analyses under a wide range of series capacitor compensations and loading conditions, time domain simulations under a pulsed torque disturbance, and fast Fourier transform (FFT) analyses for system behaviour are all performed to demonstrate the effectiveness of the proposed controller.
\end{abstract}

\section{List of symbols}

$$
\begin{array}{ll}
V, I & =\text { voltage and current } \\
R, X & =\text { resistance and reactance } \\
B & =\text { admittance } \\
E_{F D} & =\text { per unit output voltage of the exciter } \\
V_{R} & =\text { voltage regulator output } \\
T & =\text { torque } \\
a & =\text { actuator signal } \\
g & =\text { governor opening } \\
e_{c} & =\text { series capacitor voltage } \\
K_{P}, K_{I} & =\text { gains of MPI controller } \\
\omega, \theta & =\text { rotor speed and angle } \\
\delta & =\text { generator torque angle }
\end{array}
$$

\section{Subscripts}

$$
\begin{array}{ll}
d, q & =d \text {-axis and } q \text {-axis stator quantities } \\
F & =\text { field circuit } \\
D & =d \text {-axis damper } \\
Q, S & =q \text {-axis damper } \\
C & =\text { series capacitor } \\
c & =\text { shunt capacitor } \\
L & =\text { series inductor } \\
l & =\text { shunt inductor } \\
t & =\text { generator terminal } \\
b & =\text { SVC circuit } \\
H, I & =\text { high and medium pressure turbines } \\
A, B & =\text { low pressure turbines } A \text { and } B \\
X & =\text { exciter }
\end{array}
$$

\section{(C) IEE, 1993}

Paper 9522C (P6), first received 26th February 1993

The authors are with the Department of Electrical Engineering, $\mathrm{Na}$ tional Taiwan University, Taipei, Taiwan, Republic of China

\author{
Prefix \\ $\Delta \quad=$ linearised or deviation quantities \\ 1 Introduction
}

Numerous countermeasures to the subsynchronous resonance (SSR) problem such as excitation controllers [1, 2], static VAR compensators (SVC) [3-8], superconducting magnetic energy storage units [9], and others have been developed since the first two shaft failures occurred at the Mohave station in 1970 and 1971 [10]. Of these, the application of SVC for enhancing the damping of SSR will be investigated in this paper.

From a historical viewpoint, reactive power compensators such as static VAR compensators (SVC) have been applied to control bus voltage [11] and damp out low frequency oscillation mode (electromechanical mode) $[12,13]$ in a power system. The utilisation of reactive power control to damp SSR was first proposed by Putman and Ramey in 1979 [3]. Generator speed was used as a signal to modulate the reactive powers absorbed by a static VAR unit so that the torsional oscillations could be effectively suppressed. In this control scheme, although one of the unstable modes in the first benchmark model [14] can be stabilised, two other unstable critical modes still cannot be effectively controlled. Wasynczuk [4] introduced a reactive power controller which used a torsional monitor to get medium pressure turbine speed, two low pressure turbine speeds, and generator shaft speed to obtain the modal speeds to control the torsional oscillations. It showed that this control scheme was able to stabilise the unstable SSR modes of the IEEE first benchmark model system. Hammad and El-Sadek [6] presented a static VAR compensator with a main voltage regulator and an auxiliary speed signal for enhancing the damping of SSR. Owing to only utilising a proportional controller with generator speed as a feedback signal, some eigenvalues from their control scheme, though on the left half plane, were found to be very close to the imaginary axis. Balda et al. proposed an optimal output feedback design of shunt reactor controller to damp out torsional oscillations [7]. One of the major difficulties of this linear quadratic approach is how to designate the elements in the weighting matrices $Q$ and $R$ for the performance index. Recently, Wang $e t a l$. addressed a single input multiple output control scheme using both generator output current $I$ and shaft speed $\omega$ as feedback signals to modulate reactive powers of a static VAR compensator to suppress various SSR modes. Since the generator output current $I$ often varies with the operating conditions, in a practical power system, we must reset the reference value of this feedback signal $I$ to obtain an exact output feedback signal $\left(\Delta I=I-I_{\text {ref }}\right)$

IEE PROCEEDINGS-C, Vol. 140, No. 6, NOVEMBER 1993 
under the varied operating points. This is why another SIMO control scheme with fixed reference values of feedback signals will be proposed by authors to damp out torsional oscillations.

In this paper, a new SIMO static VAR controller is presented. An eigenstructure assignment technique, which was successfully used to design power system stabilisers (PSS) for damping low frequency oscillations in power systems $[15,16]$, will be applied to design this controller for suppressing various unstable torsional modes of the IEEE first benchmark model. Implementation of the designed SIMO controller via the eigenstructure assignment technique can be accomplished by a multiple proportional integral (MPI) controller with medium pressure turbine speed, two low pressure turbine speeds, and generator shaft speed as the feedback signals. Resetting of reference values of the feedback signals for different operating points is not needed under the proposed controller incorporated to the system for damping torsional oscillations. Eigenvalue analyses under a wide range of series capacitor compensations and loading conditions, time domain simulations under a pulsed torque disturbance, and fast Fourier transform (FFT) analyses for dynamic responses are all performed to demonstrate the validity of the addressed controller.

\section{System studied}

The system considered in this paper is the IEEE first benchmark model [14], of which one line diagram is shown in Fig. 1. A static VAR compensator (SVC) comprising a fixed capacitor and an inductor whose inductance is varied by adjusting the conduction angles of the

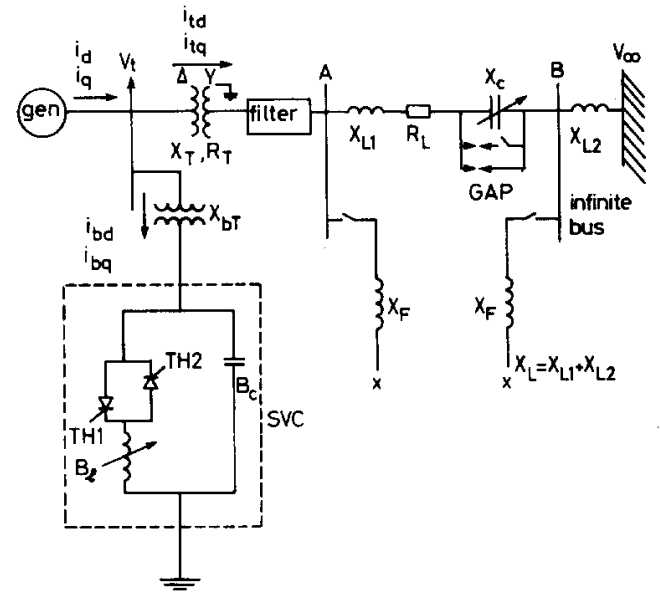

Fig. 1 IEEE First Benchmark Model with static VAR compensator included

thyristors is also incorporated to damp out SSR modes. A two time constant excitation system [1] is used. The block diagram for the static VAR compensator with supplementary control signal $U_{s}$ is shown in Fig. 2 [17].

System data $[1,14]$ used are as follows.

2.1 Inertia and stiffness constants

( $M: \mathrm{s}, K: \mathrm{pu}$ torque per rad, $D: \mathrm{pu}$ )

$$
\begin{aligned}
M_{H}=0.185794 & K_{H I}=19.303 \\
M_{I}=0.311178 & K_{I A}=34.929
\end{aligned}
$$

IEE PROCEEDINGS-C, Vol. 140, No.6, NOVEMBER 1993

$$
\begin{aligned}
& M_{A}=1.717340 \quad K_{A B}=52.038 \\
& M_{B}=1.768430 \quad K_{B G}=70.858 \\
& M_{G}=1.736990 \quad K_{G X}=2.822 \\
& M_{X}=0.068433 \\
& D_{H}=D_{I}=D_{A}=D_{B}=D_{G}=D_{X}=0.1 \\
& F_{B}=0.3 \quad T_{C H}=0.3 \mathrm{~s} \quad K_{G}=25 \\
& F_{1}=0.26 \quad T_{R H}=7.0 \mathrm{~s} \quad T_{S R}=0.2 \mathrm{~s} \\
& F_{A}=0.22 \quad T_{C o}=0.2 \mathrm{~s} \quad T_{S M}=0.3 \mathrm{~s} \\
& F_{B}=0.22
\end{aligned}
$$

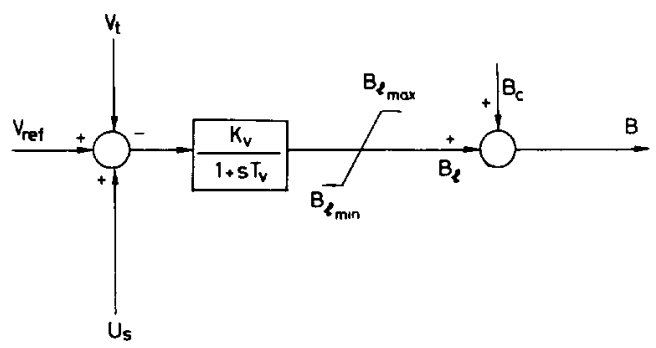

Fig. 2 Static VAR compensator with supplementary control signal $U$, included

$$
\begin{aligned}
& \text { 2.3 Exciter and voltage regulator }
\end{aligned}
$$

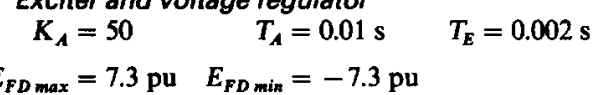

2.4 The synchronous generator ( $p u)$

$$
\begin{aligned}
& X_{d}=1.790 \quad X_{q}=1.710 \quad X_{F}=1.700 \\
& X_{D}=1.666 \quad X_{Q}=1.695 \quad X_{S}=1.825 \\
& X_{m d}=1.660 \quad X_{m q}=1.580 \quad R_{a}=0.0015 \\
& R_{F}=0.001 \quad R_{D}=0.0037 \\
& R_{S}=0.0182 \quad R_{Q}=0.0053
\end{aligned}
$$

2.5 Transformer and transmission line

$$
\begin{aligned}
& R_{T}=0.01 \mathrm{pu} \quad X_{T}=0.14 \mathrm{pu} \quad R_{L}=0.02 \mathrm{pu} \\
& X_{L}=0.56 \mathrm{pu} \quad X_{C} / X_{L}=0-100 \%
\end{aligned}
$$

$$
\begin{aligned}
& 2.6 \text { Static VAR compensator } \\
& K_{v}=50 \quad T_{v}=0.15 \mathrm{~s} \quad X_{b T}=0.08 \mathrm{pu} \\
& B_{c}=0.50 \mathrm{pu} \\
& B_{l 0}=\text { steady-state value of } B_{l}=-0.45 \mathrm{pu} \\
& B_{l_{\max }}=-0.20 \mathrm{pu} \quad B_{l_{\min }}=-0.8 \mathrm{pu} \\
& U_{\operatorname{smx}}=0.05 \mathrm{pu} \quad U_{\sin }=-0.05 \mathrm{pu}
\end{aligned}
$$

\subsection{The initial operating condition}

$$
P_{G}=0.9 \mathrm{pu} \quad V_{t}=1.05 \mathrm{pu} \quad P F=0.9 \text { (lagging) }
$$

Eigenvalues of the system without static VAR compensator are listed in the second column of Table 1. The real parts of the eigenvalues associated with the SSR modes (modes $0-5$ ) are plotted in Fig. 3 against different degrees $\left(X_{C} / X_{L}\right)$ of series compensation. It can be observed from Table 1 and Fig. 3 that damping of modes 0, 1, 3, and 4 is unsatisfactory. Thus only these modes need to be controlled by the proposed static VAR controller. 
Table 1: System eigenvalues at $P_{G}=0.9 \mathrm{pu}, P F=0.9$ legaing $V=1.05 \mathrm{pu}$ and $X_{c} / X_{L}=50 \%$

\begin{tabular}{|c|c|c|c|}
\hline Modes & Without SVC & $\begin{array}{l}\text { With SVC but } \\
\text { without controller }\end{array}$ & $\begin{array}{l}\text { With SVC and } \\
\text { proposed controller }\end{array}$ \\
\hline $\begin{array}{l}\text { Mode } 5\left(\lambda_{H}\right) \\
\text { Mode } 4\left(\lambda_{)}\right) \\
\text {Mode } 3\left(\lambda_{A}\right) \\
\text { Mode } 2\left(\lambda_{x}\right) \\
\text { Mode } 1\left(\lambda_{\theta}\right) \\
\text { Mode } 0\left(\lambda_{G}\right)\end{array}$ & $\begin{array}{r}-0.1818 \pm j 298.18 \\
-0.0231 \pm j 202.72 \\
0.7983 \pm j 160.81 \\
-0.6415 \pm j 127.07 \\
-0.1101 \pm j 99.50 \\
-0.1961 \pm j 10.02\end{array}$ & $\begin{array}{r}-0.1818 \pm j 298.18 \\
-0.0845 \pm j 202.61 \\
0.5466 \pm j 160.68 \\
-0.6380 \pm j 127.07 \\
-0.1112 \pm j 99.44 \\
-0.3501 \pm j 10.06\end{array}$ & $\begin{array}{l}-0.1821 \pm j 298.24 \\
-1.60 \pm j 207.00^{*} \\
-1.98 \pm j 159.64^{*} \\
-0.7394 \pm j 127.05 \\
-1.51 \pm j 100.10^{*} \\
-2.06 \pm j 10.00^{*}\end{array}$ \\
\hline Other modes & $\begin{array}{l}-3.3039 \\
-3.9308 \\
-0.1418 \\
-4.8391 \times j 0.2884 \\
-7.0995 \times j 590.99 \\
-6.9314 \pm j 162.49 \\
-1.8035 \\
-24.8055 \\
-32.1181 \\
-8.0871 \\
-499.9778 \\
-101.6738\end{array}$ & $\begin{array}{l}-4.7318 \\
-5.4692 \\
-0.1414 \\
-4.5410 \pm j 0.9528 \\
-5.9842 \pm j 591.64 \\
-10.7893 \pm j 163.38 \\
-2.6479 \\
-24.9711 \\
-42.2547=j 18.50 \\
-499.9747 \\
-102.9647 \\
-0.7192 \\
-0.0581 \pm j 367.62\end{array}$ & $\begin{array}{l}-5.0509 \& j 0.4655 \\
-0.1357 \\
-3.2315 \pm j 0.9110 \\
-5.8915 \pm j 591.71 \\
-6.0949 \pm j 165.64 \\
-5.1275 \\
-25.0230 \\
-37.7778 \propto j 16.42 \\
-499.9743 \\
-102.5753 \\
-0.4746 \\
-2.4830 \pm j 363.72\end{array}$ \\
\hline
\end{tabular}

- Denotes the desired eigenvalues for controller design.

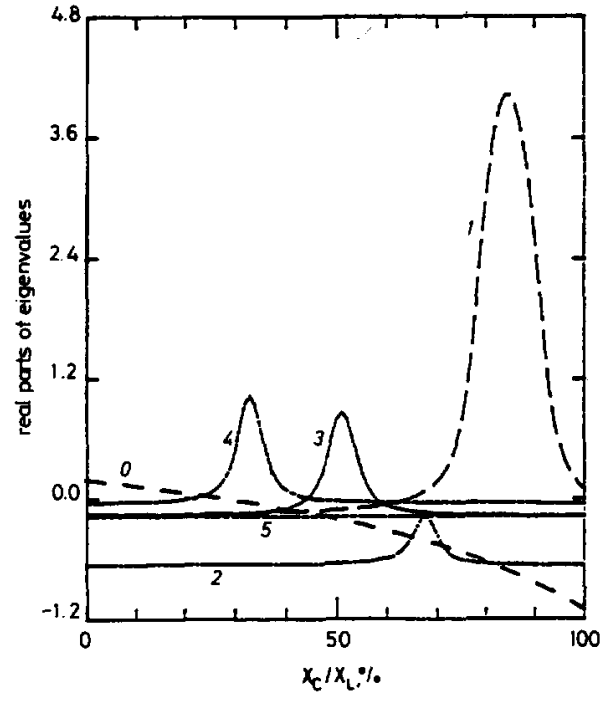

Fig. 3 Real parts of SSR mode eigenvalues as function of $X_{C} / X_{L}$ system without SVC, $P_{\theta}=0.9 \mathrm{pu}$

...... mode 0 mode 3

- - mode 4

\section{Eigenatructure sesignment technique}

In this Section, the eigenstructure assignment technique using output feedback is reviewed $[18,19]$ to design a SIMO static VAR controller in the next Section.

We consider a linear time invariant system described by equations

$$
\begin{aligned}
& \dot{X}(t)=A X(t)+B U(t) \\
& Y(t)=C X(t)
\end{aligned}
$$

where $X \in R^{n}, U \in R^{m}, Y \in R^{r}$ are called the state, control and output, respectively; $A, B$, and $C$ are real constant matrices of appropriate dimensions.

If the above system is controllable, and if the rank of $C$ is $r$, a linear feedback control law of the form

$$
U(t)=F Y(t)
$$

464 can always be found and the theorem $[18,19]$ is abbreviated as follows.

\subsection{Theorem}

Given the controllable and observable system described by eqns. 1 and 2 , and the assumptions that the matrices $B$ and $C$ are of full rank, then $\max (m, r)$ closed loop eigenvalues can be assigned, and $\max (m, r)$ eigenvectors can be partially assigned with $\min (m, r)$ entires in each vector arbitrarily chosen using the output feedback gain.

Assuming that the desired eigenstructure to be assigned is $\lambda_{d_{1}}$ and $v_{d_{1}}, i=1,2, \ldots, r$, the best assignable eigenvector $v_{a_{1}}[18]$ is defined as

$$
v_{a_{t}} \triangleq L_{i}\left(L_{l}^{T} L_{l}\right)^{-1} L_{l}^{T} v_{d t}
$$

where

$$
L_{i} \triangleq\left(\lambda_{d_{i}} I-A\right)^{-1} B
$$

Once the $\lambda_{d_{1}}$ and $v_{d_{1}}$ are assigned, the best possible assignable eigenvector $v_{a_{i}}$ can be derived from eqn. 3 .

In the power system, we are only interested in some special entry such as $\Delta \omega$ of the eigenvector. Thus the design problem will become a partial specification condition.

In such a case, we can reorder the desirable entries to the leading position of $v_{d l}$, such that

$$
v_{d_{i}} \rightarrow\left[\begin{array}{l}
z_{d_{i}} \\
v_{x_{i}}
\end{array}\right]
$$

where $z_{d_{1}}$ is the vector of assigned entries, and $v_{x_{1}}$ represents the vector of unassigned parts.

We also reorder the rows of $L_{i}$ to conform with the reordered components of $v_{d_{1}}$, i.e.

$$
L_{i} \rightarrow\left[\begin{array}{l}
D_{i} \\
L_{x_{i}}
\end{array}\right]
$$

Then we have

$$
v_{a_{i}}=L_{i}\left(D_{i}^{T} D_{i}\right)^{-1} D_{i}^{T} z_{d_{i}}
$$

Here, $v_{a}$ is the best possible assignable eigenvector concerning $\lambda_{d_{1}}$ and $v_{d_{1}}$

Now as shown [18] by a similarity transformation and some manipulations, we can obtain the following equation which holds for each desired eigenvalue, achiev- 
able eigenvector pair $\lambda_{d_{1}}, v_{a !}$ :

$$
\left(A_{1}+F C\right) v_{a_{1}}=\lambda_{d i} z_{a_{i}}
$$

where $z_{a_{1}}$ is the specified part of $v_{a_{i}}$, and $A_{1}$ is the submatrix of $A$ conforming with $z_{a}$.

Thus for all desired eigenvalue, achievable eigenvector pairs, we get the following identity in condensed form [18]:

$$
\left(A_{1}+F C\right) V_{a}=Z
$$

where

$$
\begin{aligned}
& V_{a}=\left[\begin{array}{llll}
v_{a 1} & v_{a_{2}} & \cdots & v_{a_{r}}
\end{array}\right] \quad(n \times r) \\
& Z=\left[\begin{array}{lllll}
\lambda_{d_{1}} z_{a_{1}} & \lambda_{d_{2}} z_{a_{2}} & \cdots & \lambda_{d_{r}} z_{a_{2}}
\end{array}\right] \quad(m \times r)
\end{aligned}
$$

If $C V_{a}$ is nonsingular, there exists an inverse of $C V_{a}$. Then the feedback gain matrix $F$ can be obtained from eqn. 9 as

$$
F=\left(Z-A_{1} V_{a}\right)\left(C V_{a}\right)^{-1}
$$

\section{Design of a single input multiple output (SIMO) static VAR controller}

To improve the damping for modes $0,1,3$, and 4 , a static VAR compensator whose block diagram is shown in Fig. 2 (without supplementary control signal $U_{3}$ ) is incorporated into the system. The resulting system eigenvalues are listed in the third column of Table 1, and the real parts of the SSR mode eigenvalues for different degrees of series compensation are plotted in Fig. 4. An observation of

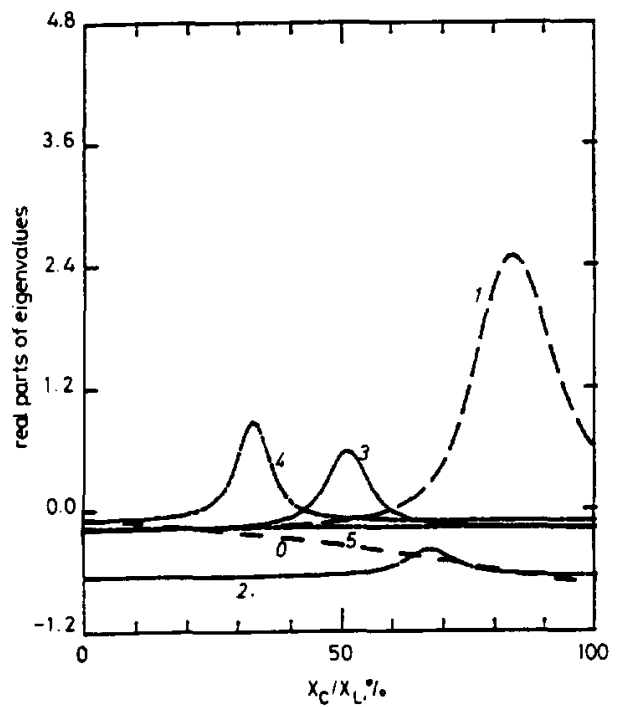

Fig. 4 Real parts of SSR mode eigenvalues as function of $X_{c} / X_{L}$ system with SVC but without controller, $P_{G}=0.9 \mathrm{pu}$

...-.- mode 0 mode 3

Table 1 and Fig. 4 reveals that the static VAR compensator alone is still insufficient to enhance the damping of modes $0,1,3$, and 4 . A supplementary control signal is therefore required for this purpose. An SIMO static VAR controller is presented to generate this signal. To design this controller via the eigenstructure assignment technique described in the previous Section, the system studied under the initial operating condition, and the

IEE PROCEEDINGS-C, Vol.140, No. 6, NOVEMBER 1993 degree $X_{C} / X_{L}=50 \%$ of series compensation, is first linearised. The state equations of the linearised system can be written as

$$
\begin{aligned}
& \dot{X}(t)=A X(t)+B U(t) \\
& Y(t)=C X(t)
\end{aligned}
$$

where

$$
\begin{aligned}
X= & {\left[\Delta \omega, \Delta \delta, \Delta \omega_{H}, \Delta \theta_{H}, \Delta \omega_{I}, \Delta \theta_{I}, \Delta \omega_{A}, \Delta \theta_{A}, \Delta \omega_{B},\right.} \\
& \Delta \theta_{B}, \Delta \omega_{X}, \Delta \theta_{X}, \Delta T_{H}, \Delta T_{I}, \Delta T_{A}, \Delta a, \Delta g, \Delta e_{c d}, \\
& \Delta e_{c q}, \Delta i_{d}, \Delta i_{F}, \Delta i_{D}, \Delta i_{q}, \Delta i_{Q}, \Delta i_{S}, \Delta V_{R}, \Delta E_{F D}, \\
& \left.\Delta i_{b d}, \Delta i_{b q}, \Delta B_{i}\right]^{T}
\end{aligned}
$$

is the state vector

$$
Y=\left[\Delta \omega, \Delta \delta, \Delta \omega_{I}, \Delta \theta_{I}, \Delta \omega_{A}, \Delta \theta_{A}, \Delta \omega_{B}, \Delta \theta_{B}\right]^{T}
$$

is the output vector, $U=U_{\text {, is }}$ the supplementary control signal, and $A, B$, and $C$ are the real constant matrices.

An examination of the necessary conditions of the theorem in the previous Section for the proposed controller design indicates that the above system is both controllable and observable, and the matrices $B$ and $C$ are of full rank. Then the eigenstructure assignment technqiue can be used to determine the feedback gains of the introduced controller.

The configuration of the SIMO static VAR controller is shown in Fig. 5. From it, we understand that the

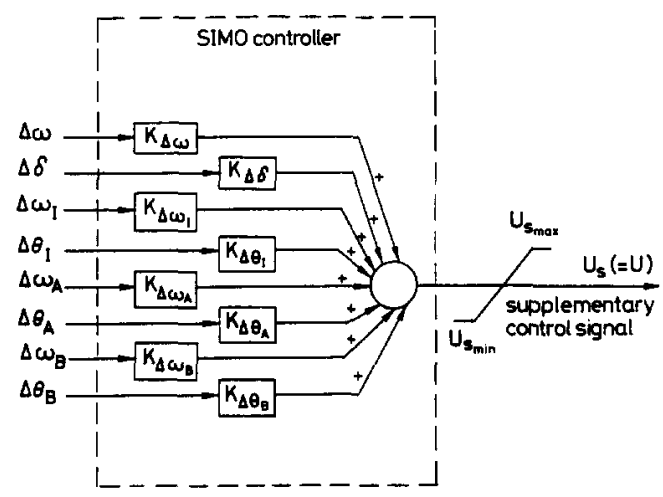

Fig. 5 Configuration of SIMO static VAR controller

system studied with this controller is a single input eight output system. Then based on the theorem in the previous Section, we can assign eight closed loop eigenvalues, and their associated eigenvectors can be partially assigned with one element in each vector arbitrarily specified. The assigned eigenvalues and eigenvectors are described as follows for determining the feedback gains.

In this paper, the prespecified eignvalues of the torsional modes are placed at

$$
\begin{array}{ll}
\lambda_{1,2}=-2.06 \pm j 10.00 & (\text { mode } 0) \\
\lambda_{3,4}=-1.51 \pm j 100.10 & (\text { mode } 1) \\
\lambda_{5,6}=-1.98 \pm j 159.64 & (\text { mode } 3) \\
\lambda_{7,8}=-1.60 \pm j 207.00 & \text { (mode } 4)
\end{array}
$$

As for the desired element of each eigenvector corresponding to each assigned eigenvalue, we choose the speed deviation state $\Delta \omega$ as the element to be specified, and this element can always be set to the value of 1.0 for all 
desired eigenvectors corresponding to all prespecified eigenvalues.

Given the above eigenstructure (eigenvalue assignment and eigenvector assignment), the feedback gain matrix

$$
F=\left[K_{\Delta \omega}, K_{\Delta \delta}, K_{\Delta \omega_{1}}, K_{\Delta \theta_{I}}, K_{\Delta \omega_{1}}, K_{\Delta \theta_{1}}, K_{\Delta \omega_{g}}, K_{\Delta \theta_{g}}\right]
$$

can be figured out by eqn. 10 , and then

$$
\begin{aligned}
F= & {[9.0036,-7.3146,-0.8785,2.3297,-0.0620,} \\
& 0.6212,3.0905,4.4116]
\end{aligned}
$$

The values of the prespecified eigenvalues are chosen arbitrarily according to the expectation of the damping of the SSR modes, while that of the element $\Delta \omega$ of each eigenvector corresponding to each prespecified eigenvalue is fixed (equal to 1.0 ) for simplicity. If the feedback gain values are outside a reasonable range, the assigned eigenvalues must be adjusted until the feedback gain values are located within a suitable range.

A relevant comment on the implementation of the SIMO controller whose configuration is shown in Fig. 5

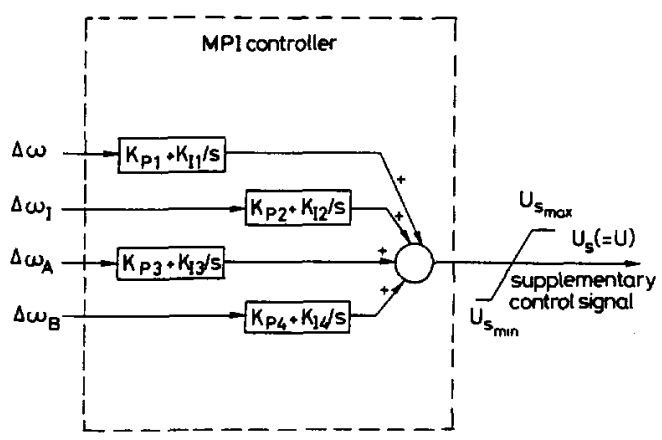

Fig. 6 Implementation of Fig. 5

is that such a controller can be implemented as a multiple proportional integral (MPI) controller, shown in Fig. 6 , using the medium pressure turbine deviation speed $\left(\Delta \omega_{I}=\omega_{I}-1\right)$, the two low pressure turbine deviation speeds $\left(\Delta \omega_{A}=\omega_{A}-1\right.$ and $\left.\Delta \omega_{B}=\omega_{B}-1\right)$, and the generator shaft deviation speed $(\Delta \omega=\omega-1)$ as the inputs of the MPI controller. This is due to the fact that the angle deviation is the time integral of the speed deviation. Comparison of Fig. 5 with Fig. 6 yields

$$
\begin{array}{ll}
K_{P 1}=K_{\Delta \omega} & K_{I 1}=K_{\Delta \theta} \\
K_{P 2}=K_{\Delta \omega t} & K_{12}=K_{\Delta \theta_{I}} \\
K_{P 3}=K_{\Delta \omega \Delta} & K_{I 3}=K_{\Delta \theta_{A}} \\
K_{P 4}=K_{\Delta \omega z} & K_{14}=K_{\Delta \theta_{3}}
\end{array}
$$

Eqns. 13-16 set up the equivalence of the SIMO controller to the MPI controller. Meanwhile, it is worth noting that the reference values of the feedback signals $\left(\omega_{I}, \omega_{A}\right.$ $\omega_{\mathrm{B}}$, and $\omega$ ) are always fixed at $1.0 \mathrm{pu}$ for any operating point. Therefore there will be no need to reset reference values of these feedback signals for different operating conditions in this case.

To ensure the successful operation of the proposed controller in a real power system, two things need to be done. The first is the problem of noisy signals. Noisy signals come from the feedback signal path and can give rise to false action in the controller, even though the system is normal. This false control action will make the modulations in the inductive susceptance of the SVC. As a result, the variations in susceptance cause undesirable system oscillations. To avoid this, proper filtering devices inserted into the feedback signal paths are required. The second is problems in the accuracy of feedback speeds. Since deviations in speed across different turbine spindles are small, fast and precise speed sensors are therefore needed such that the deviations in each feedback speed can be quickly and accurately sensed and imported into the controller. If not, improvements in damping SSR using the suggested controller will be restrained. In this paper, both the accuracy of the feedback signals achieved by the measurement devices and noisy signals eliminated by filtering devices are assumed so that the basic characteristics of the proposed controller in damping torsional oscillations can be explored with ease.

\section{Eigenvalue analyses}

Eigenvalues for the system with SVC and the proposed controller are listed in the fourth column of Table 1 . The real parts of the SSR mode eigenvalues for different degrees of series compensation are plotted in Fig. 7. It is found that the prespecified eigenvalues for modes $0,1,3$, and 4 can be assigned exactly, and damping for all SSR modes is satisfactory for any $X_{C} / X_{L}$ value despite the fact that the controller is designed for $X_{C} / X_{L}=50 \%$.

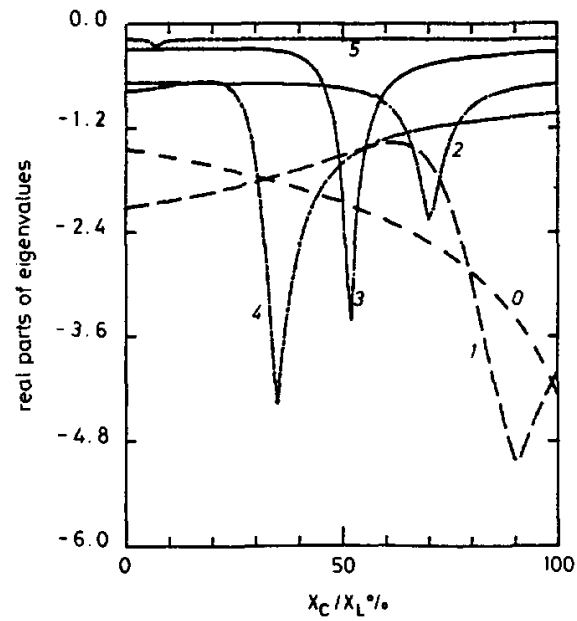

Fig. 7 Real parts of SSR mode eigenvalues as function of $X_{d} / X_{L}$ system with SVC and proposed controlier, $P_{c}=0.9 \mathrm{pu}$ -...... mode 0

- - - mode

-... mode 2

- mode 3

mode 5

To examine the control ability of the suggested controller at other loading conditions, the real parts of the SSR mode eigenvalues are plotted in Fig. 8 against the different values of the loading condition $P_{G}$. It can be concluded from this Figure that the proposed controller, though designed at the loading condition $P_{G}=0.9 \mathrm{pu}$, is effective in damping all SSR modes for a wide range of loading conditions.

\section{Time domain simulations}

To illustrate the effectiveness of the addressed controller for damping torsional oscillations under disturbance con-

IEE PROCEEDINGS-C, Vol. 140, NO. 6, NOVEMBER 1993 
ditions, digital computer simulations are performed for the system, with $X_{C} / X_{L}=50 \%$ and $P_{G}=0.9 \mathrm{pu}$, subject to a pulsed torque disturbance $\Delta T_{e}$ of $10 \%$ for $0.1 \mathrm{~s}$. Dynamic response of the system without SVC, and with SVC and the proposed controller are shown in Figs. 9 and 10, respectively. The response curves in Fig. 9 and 10

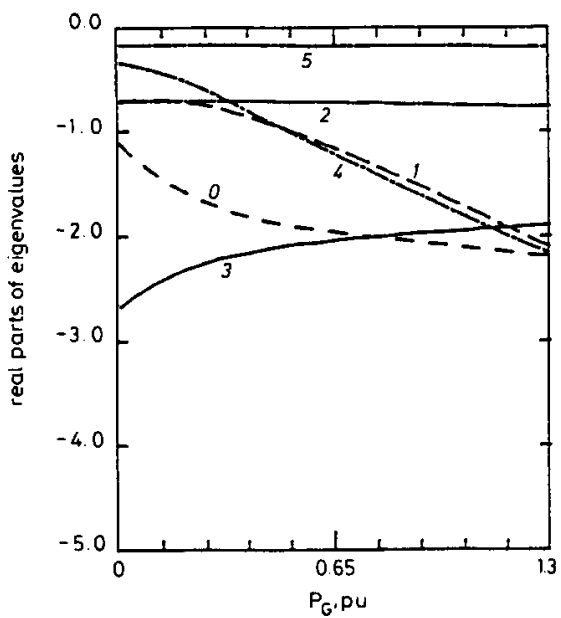

Fig. 8 Real parts of SSR mode eigenvalues as function of $P_{G}$ system with SVC and proposed controller, $X_{d} / X_{L}=50 \%$

-...... mode 0

- - - mode 1

mode 2

- mode 3

--.- made 4

show that unstable torsional oscillations can be effectively stabilised and significant improvements in dynamic performance can be obtained by the proposed SVC controller.
7

\section{Fast Fourier transform (FFT) analyses}

Though time domain simulations can give the dynamic behaviour of the system subject to disturbance, it is difficult to look into the degree of SSR mode components contained in the system behaviour. A fast Fourier transform (FFT) algorithm is applied to do this. By using this algorithm, the frequency spectrum of the dynamic responses of $\Delta \omega$ plotted in Fig. $9 d$ and Fig. 10d are shown in Fig. 11. It can be found that the SSR mode components (modes $0,1,3$, and 4 ) in dynamic response can certainly be reduced significantly by the proposed controller.

\section{Conclusions}

A new approach of designing a single input multiple output (SIMO) static VAR controller via the eigenstructure assignment technique for damping torsional oscillations has been presented. The introduced controller can be implemened by a multiple proportional integral (MPI) controller. It is not found necessary to reset reference values of feedback signals for different operating conditions under the suggested controller incorporated to the system. The excellent performances of the proposed controller in damping SSR are also demonstrated by eigenvalue analyses, time domain simulations, and fast Fourier transform (FFT) analyses.

Successful operation of the suggested approach for damping SSR requires fast and precise speed measurement devices and proper filtering devices in the feedback signal paths to eliminate the noisy signals and obtain accurate feedback signals for a practical power system.

Up until now, the effectiveness has been tested by simulations only. Further examination using experimental results is a future project.
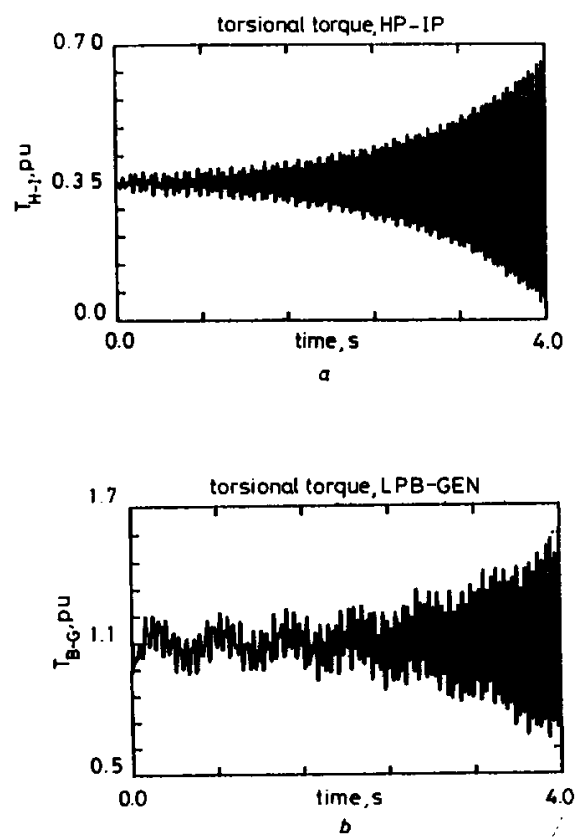

Fig. 9 Dynamic responses for system without SVC $P_{0}=0.9 \mathrm{pu}$

IEE PROCEEDINGS.C, Vol. 140, No. 6, NOVEMBER 1993
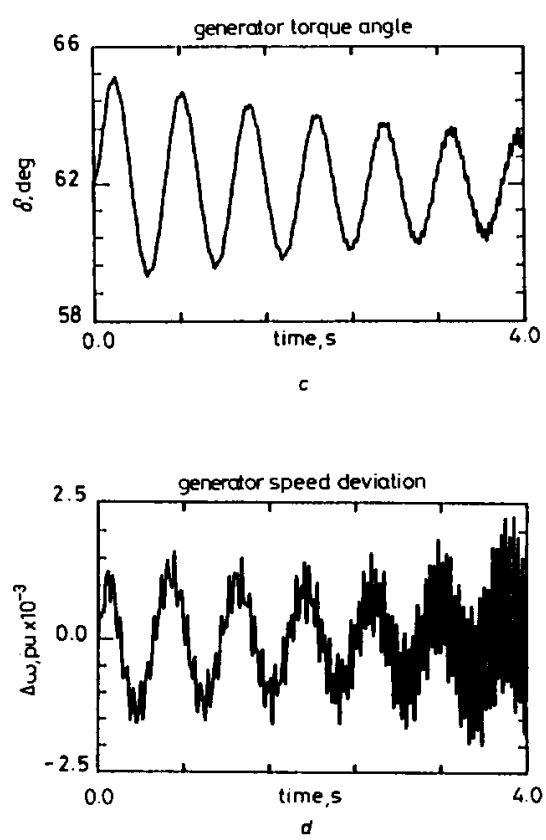

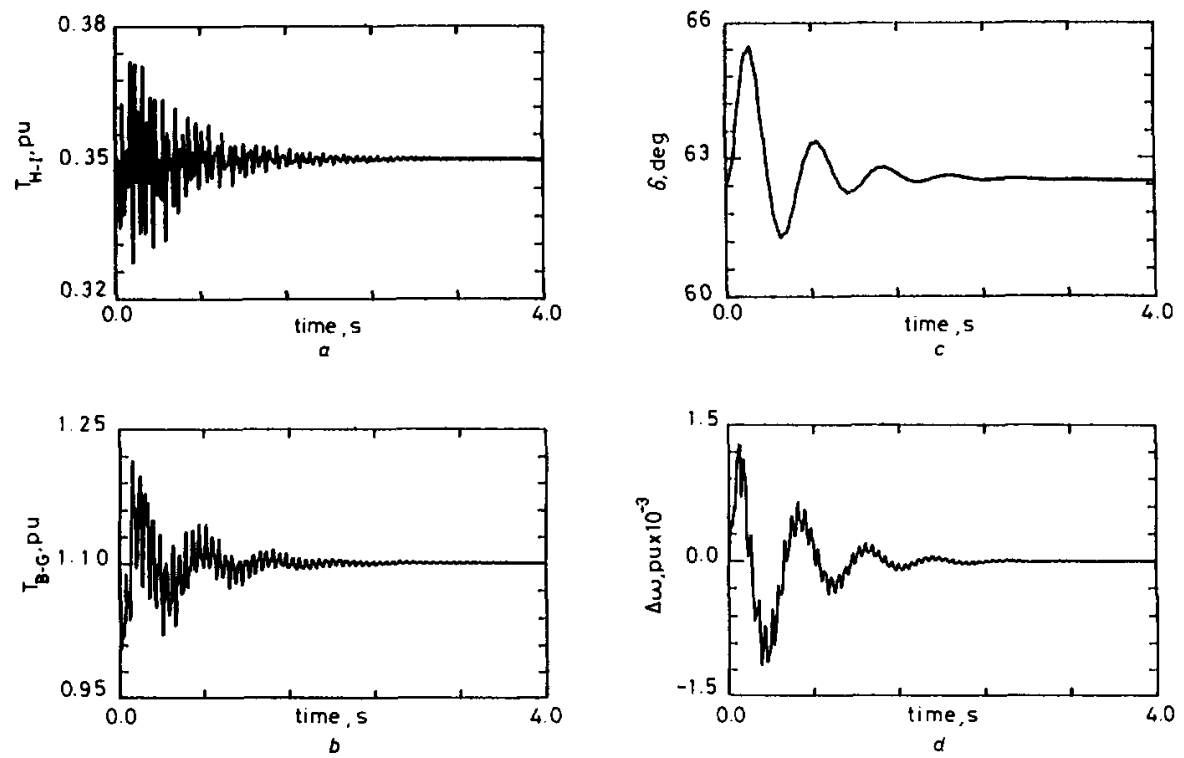

Fig. 10 Dynamic responses for system with $S V C$ and proposed controller $P_{G}=0.9 \mathrm{pu}$

$a$ torsional torque, HP-IP $b$ torsional torque, LPB-GEN $c$ generator torque angle $d$ generator speed deviation

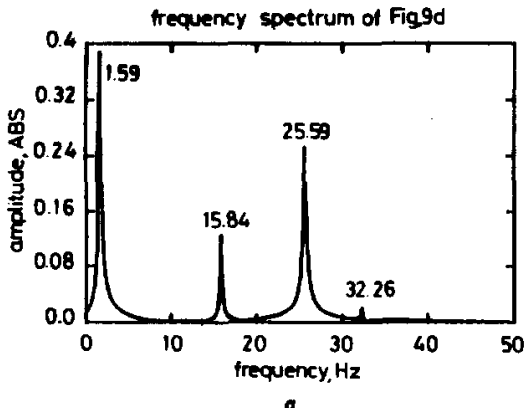

Fig. 11 Frequency spectra of $\Delta \omega$ plotted in Fig. 9d and Fig. 10d

\section{References}

1 YU, Y.N.: 'Electric power system dynamics' (Academic Press, New York, 1983)

2 YAN, A., and YU, Y.N.: 'Multi-mode stabilisation of torsional oscillations using output feedback excitation control, IEEE Trans., 1982, PAS-101, pp. 1245-1253

3 PUTMAN, T.H and RAMEY, D.G.: 'A modulated inductance stabilizer for power system subject to subsynchronous resonance'. Pre-
sented at the International Symposium on Static VAR sented at the International Sym
compensation, Montreal, Canada, 1979

4 WASYNCZUK, O.: 'Damping subsynchronous resonance using reactive power control', IEEE Trans., 1981, PAS-100, pp. 1096-1104 BOWLER, C.E.J.: 'Discussion on [4]', IEEE Trans., 1981, PAS-100, p. 1103

6 HAMMAD, A.E., and EL-SADEK, M.: 'Application of a thyristor controlled VAR compensator for damping subsynchronous oscillations in power systems', IEEE Trans., 1984, PAS-103, pp. 198-212

7 BALDA, J.C., EITELBERG, E., and HARLEY, R.G.: 'Optimal output feedback design of a shunt rector controller for damping torsional oscillations', Electr. Power Syst. Res., 1986, 10, pp. 25-33

8 WANG, L. and HSU, Y.Y.: 'Damping of subsynchronous resonance using excitation controllers and static VAR compensators' a comparative study', IEEE Trans, 1988, EC-3, pp. 6-13

9 LEE, Y.S., and WU, C.J.: 'Application of superconducting magnetic energy storage unit on damping of turbogenerator subsynchronous oscillation', IEE Proc. C, 1991, 138, pp. 419-426

10 HALL, M.C., and HODGES, D.A.: 'Experience with $500 \mathrm{kV}$ sub-

468

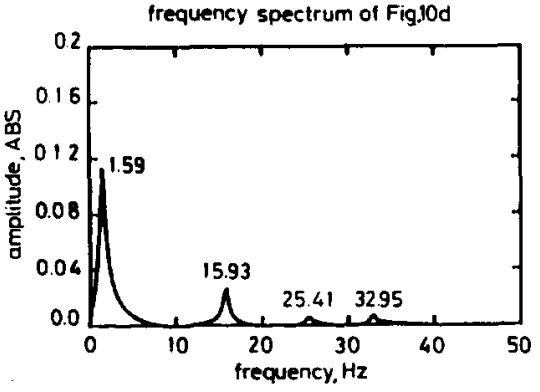

synchronous resonance and resulting turbine generator shaft damage at Mohave generating station'. IEEE, No. 76, New York 1976 , pp. 22-29

11 HAUTH, R.L., HUMANN, T., and NEWELL, R.J.: 'Application of a static VAR system to regulate system voltage in Western Nebraska', IEEE Trans., 1978, PAS-97, pp. 1955-1964

12 KAPOOR, S.C.: 'Dynamic stability of long transmission systems with static compensators and synchronous machines', IEEE Trans., 1979, PAS-98, pp. 124-134

13 BYERLY, R.T., POZNANIAK, D.T., and TAYLOR, E.R. Jr.: 'Static reactive compensation for power transmission systems', IEEE Trans., 1982, PAS-101, pp. 3997-4005

14 IEEE SSR Working Group: 'First benchmark model for computer simulation of subsynchronous resonance', IEEE Trans., 1977, PAS-96, pp. 1565-1572

15 HUANG, P.H., and HSU, Y.Y.: 'An output feedback controller for a synchronous generator', IEEE Trans., 1990, AES-26, pp. 337-344

16 HUANG, P.H., and HSU, Y.Y.: 'Eigenstructure assignment in a longitudinal power system via excitation control', IEEE Trans. 1990, PWRS-5, pp. 96-102

17 HSU, Y.Y., and WU, C.J.: 'Design of PID static VAR controllers for the damping of subsynchronous oscillations', IEEE Trans., 1988, EC-3, pp. 210-216

18 ANDRY, A.N., SHAPIRO, E.Y., and CHUNG, I.C.: 'Eigenstructure assignment for linear systems', IEEE Trans., 1983, AES-19, pp. 711-729

19 SRINATHKUMAR, S.: 'Eigenvalue/eigenvector assignment using output feedback', IEEE Trans., 1978, AC-23, pp. 79-81

IEE PROCEEDINGS-C, Vol. 140, No. 6, NOVEMBER I993 